\title{
Global Canny algorithm based on Canny edge detector framework in magnetic resonance imaging
}

\author{
Tengku Ahmad Iskandar Tengku Alang a , Tan Tian Swee b, ${ }^{*}$, Tan Jia Hou a, Leong Kah Meng a, \\ Sameen Ahmed Malik a, Muhammad Amir As'ari a, Adil Hussein ${ }^{c}$, Azhany Yaakub c, Hum Yan \\ Chai ${ }^{d}$, Juhara Haron ${ }^{e}$
}

a Department of Biotechnology and Medical Engineering, Faculty of Biosciences and Medical Engineering, Universiti Teknologi Malaysia, 81310 UTM Johor Bahru, Johor, Malaysia

b Medical Implant Technology Group (MediTEG), Frontier Materials Research Alliance, Faculty of Biosciences and Medical Engineering, Universiti Teknologi Malaysia, 81310 UTM Johor Bahru, Johor, Malaysia

- Department of Ophthalmology, School of Medical Sciences, Universiti Sains Malaysia, 16150 Kubang Kerian, Kelantan, Malaysia

${ }^{d}$ Lee Kong Chian Faculty of Engineering and Science, Department of Mechatronics and BioMedical Engineering, Universiti Tunku Abdul Rahman, 46200 Petaling Jaya, Selangor, Malaysia

e Department of Radiology, School of Medical Sciences, Universiti Sains Malaysia, 16150 Kubang Kerian, Kelantan, Malaysia

*Corresponding author: tantswee@biomedical.utm.my

\section{Article history}

Received 28 September 2017

Accepted 7 December 2017

\section{Graphical abstract}

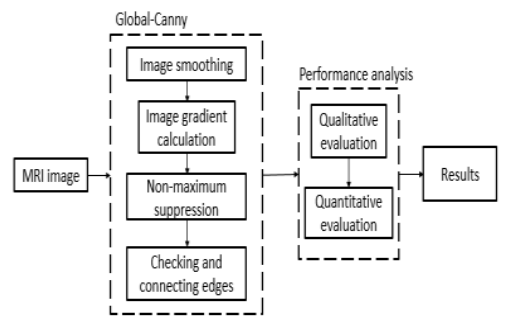

\begin{abstract}
Magnetic resonance imaging is an important modality in the diagnosis and pathology detection. Edge detection is used for image segmentation and feature extraction as part of the medical image analysis. There is no ideal and universal algorithm which performs perfectly under all conditions. Conventional Canny edge detector is widely used as it produces excellent edge detection results, however the filter comprises Gaussian smoothing element that may significantly blurring the edges. In this paper, we propose the use of customized non-local means into the Canny edge detector to replace the Gaussian smoothing in the conventional Canny edge detector in order to effectively remove Rician noise while preserving edges in Magnetic resonance image of an internal organ. The result shows that our method can yield better edge detection than conventional method, with minimal false edge detection. The proposed method undergoes several attempts of parameter adjustment to detect true edges successfully using optimal parameter setting.
\end{abstract}

Keywords: Edge detection, Non-local means, Canny edge detector, Magnetic resonance images, Segmentation.

\section{INTRODUCTION}

Edge detection is one of the most important feature in the application of image processing, computer vision and machine vision (Selvakumar \& Hariganesh, 2016; X. Jia, 2010; Zhang et al., 2015; Benson et al., 2003). The purpose is to extract information of boundaries of the object within image by detecting discontinuities or abrupt changes in brightness level. Edges provide boundaries between different regions in the image. The boundaries obtained are useful to recognize objects, such as for segmentation and matching purpose (Voorhees \& Poggio, 1987; H. Y. Chai, Wee, Swee, \& Hussain, 2011). The ideal edge detection algorithm will produce a set of connected curves that indicate boundaries of objects. Since edge detection have been used widely in many applications, it is important to design an effective and efficient edge detector as it will directly influence the image analysis. Such examples can be found in the enhancement of noisy images, namely satellite images (Gupta, 2016; Jena et al., 2015) and it has taken a great deal of attention when it comes to medical images such as MRI (Aslam et al., 2015; Giuliani, 2012; Muthukrishnan \& Radha, 2011), ultrasound (H. Chai et al., 2012; Nikolic et al., 2016), CT (Bandyopadhyay, 2012; Punarselvam
\& Suresh, 2011) and X-ray images (D. C. et al., 2012; Lakhani et al., 2016). It is also useful in monitoring the moving object in real-time surveillance and visual tracking (Anupam Mukherjee \& Debaditya Kundu, 2013; Karamiani \& Farajzadeh, 2014; Khadse \& Kale, 2016). The use of edge detection in the road mapping analysis (Qiu et al., 2016; Sirmacek \& Unsalan, 2010) benefiting the road user to deal with the various road and its boundaries. Other application of edge detection includes the detection and recognition of the text from the complex background (Pietikainen \& Okun, 2001; Yu et al., 2015).

The existing edge detectors are mostly designed on the basis of spatial domain detection that utilize gradient and Laplacian information to execute high pass filtering (Dhankhar \& Sahu, 2013; Lakshmi \& Sankaranarayanan, 2010; Pal \& Pal, 1993; Savant, 2014). Examples of gradient-based algorithms are Sobel, Prewitt, Roberts and the Laplacian of Gaussian (LoG) algorithms (Huertas \& Medioni, 1986). These methods are known to be straightforward and fast where a simple convolution operation involved between small convolution mask and the input, in order to detect gradient in the image. On top of that, it is simple to be implemented. This simplicity raised from the "single focus" approach properties of edge enhancement, but lack of smoothing operation. Hence, the gradient-based edge detectors are 
very sensitive to noise, inaccurate and imperfect in engineering applications (Wang \& Fan, 2009).

Canny edge detection is the most well-known Laplacian-based edge detector. This method is the most favorable to detect edges corrupted by noise. Canny (1986) uses a multistage algorithm to detect a wide range of edges in images. Through this technique, he defines three optimal criteria as guidelines for an effective result. First, the detector must minimize the probability of false edges caused by noise, as well as missing real edges. Second, the edges detected must be as close as possible to the true edges. Third, the detector must return one point only for each true edge point. In summary, the criteria presented by Canny filter are good detection, good localization and single response (Canny, 1986).

The performance of Canny edge detection is outstanding among other common filters in most cases, yet there is always room for improvement (Pellegrino et al., 2004; H. Y. Chai, Wee, Swee, \& Hussain, 2011). Presently, some researchers offered many improved version of Canny filter. Li et al. (2009) proposed optimization on the gradient level calculation operator and automatization of edge detection method using Otsu thresholding. The results showed good edge detection achieved to some extent. Agaian et al. (2009) introduced optimization of gradient kernel by employing Nercessian's generalized kernels of derivative approximation (Agaian et al., 2009; Nercessian et al., 2009). The results are effective in detecting branch edges in the application of asphalt concrete detection as compared to traditional Canny algorithm. Hou et al. (2009) proposed the histogram-based fuzzy $c$-means clustering algorithm to modify the original Canny method. The results showed good detection on the application of road surface distress image. In common situation, Gaussian smoothing is used to reduce the sensitivity to noise before applying Canny edge detection.

Recently, there are numerous literature that proposing different technique to detect and extract edges in the noisy medical images. This includes the edge detection speckle-noise corrupted of ultrasound images (H. Chai et al., 2012; Nikolic et al., 2016), the Gaussian noise of CT (Bandyopadhyay, 2012; Punarselvam \& Suresh, 2011) and Xray images (D.C et al., 2012; Lakhani et al., 2016), but no recent works had been done concerning to the edge detection in the Riciannoise corrupted of MR images. Original Canny edge detection method is not suitable to remove Rician noise in MR images because Gaussian filter cannot remove Rician noise effectively. Hence, noise will be perceived as the detected edges.

In this paper, an algorithm for edge detection in MR images is proposed. The first objective of this study is to reconstruct the conventional Canny edge detector by replacing Gaussian smoothing element with non-local means filter in order to remove Rician noise and to enhance the edge in MR image phantom. The second objective is to evaluate the effectiveness of the proposed method as compared to other existed algorithm. The proposed algorithm is designed on the basis of Canny edge detection algorithm. Non-local means (NLM) filter is proposed instead of Gaussian filter.

The main contribution of this work is that a new edge detection method was proposed combining the non-local means filter and Canny operator. The implementation of this technique may assist researchers and physicists in extracting the image features, expecially for MRI images. A practical quality comparison conducted between existing and the proposed techniques can provide some guidance in deciding the best technique for any specific application.

\section{MATERIALS AND METHOD}

\section{Noise modelling}

MR images are contaminated by Rician noise. This kind of noise arises from complex Gaussian noise in the original frequency domain measurements which was obtained from the raw data. Usually, magnitude image will be used for visualization and analysis. This is done by taking inverse Fourier transform of the raw data. A nonlinear mapping between pixels involved, thus the noise ditribution is no longer Gaussian.
The noise-free image pixel intensity is denoted by $A$ while the current measure pixel intensity is $M$. Mathematically, the Rician probability density function for the contaminated image intensity is given by

$$
i(M)=\frac{M}{\sigma_{n}^{2}} \exp \left(-\frac{M^{2}+A^{2}}{2 \sigma_{n}^{2}}\right) I_{0}\left(\frac{A \cdot M}{\sigma_{n}^{2}}\right)
$$

where $A$ is the underlying true intensity, $\sigma$ is the standard deviation of the noise, and $I_{0}$ is the modified zeroth order Bessel function of the first kind (Rumheller, 1993; Gudbjartsson \& Patz, 1995; Sijbers et al., 1998).

\section{Basic concept of traditional Canny edge detector}

A. Gaussian filtering

The first and foremost step in Canny edge detector is to filter the image by employing the first order of 2-D Gaussian function in any direction. This step was done to minimize the influence of noise in the edge detection that may disrupt the true optimum detection results. Usually, the filter mask of $5 \times 5$ size is used to be convolved with the image. The general equation of Gaussian filter can be defined as follows:

$$
G(x, y)=\frac{1}{2 \pi \sigma^{2}} \exp \left[-\frac{x^{2}+y^{2}}{2 \sigma^{2}}\right]
$$

The key for successful implementation of Gaussian filtering is however depends on the Gaussian kernel size. The larger the size, the lower the sensitivity to noise. The lower the size, the longer the time duration for the filtering process to be done. In most of the cases, the use of $5 \times 5$ kernel size showed good performance, but this will also vary depending on specific situations.

\section{B. Image gradient calculation}

The second step is to find the image gradient in magnitude and direction. The edges should be marked in the location that have large magnitude gradient. Canny edge detection algorithm adopts limited difference of $2 \times 2$ neighboring area to compute the magnitude and the direction of image gradient, vertically and horizontally (J. Jia, 2009). The approximation for the first order partial derivative can be obtained from these following formulas:

$$
\begin{aligned}
& E_{x}[i, j] \\
& =I(i+1, j)-I(i, j)+I(i+1, j+1)-I(i, j+1) / 2 \\
& E_{y}[i, j] \\
& =I(i, j+1)-I(i, j)+I(i+1, j+1)-I(i+1, j) / 2
\end{aligned}
$$

Therefore, the template operator that can be used to calculate image gradient can be written as follows:

$$
\begin{gathered}
G_{x}=\left(\begin{array}{ll}
-1 & 1 \\
-1 & 1
\end{array}\right) \\
G_{y}=\left(\begin{array}{cr}
1 & 1 \\
-1 & -1
\end{array}\right)
\end{gathered}
$$

Then, the magnitude and direction of image gradient can be computed using following equation. The image gradient magnitude is:

$$
\|M(i, j)\|=\sqrt{E_{x}[i, j]^{2}+E_{y}[i, j]^{2}}
$$

The direction of image gradient is

$$
\theta(i, j)=\arctan \left[E_{y}[i, j] / E_{x}[i, j]\right]
$$

\section{Non-maximum supression}

The next step is to execute non-maximum suppression. It should be only local maxima need to be marked as edges, so that we can 
accurately position edges. The process will ensure that each edge is one pixel width. Canny algorithm uses $3 \times 3$ neighboring area which consists of eight directions to execute interpolation to the gradient magnitude along gradient's direction. Through this interpolation, the gradient direction $\theta$ is rounded to the nearest $45^{\circ}$, subsequently the current pixel of the gradient magnitude is being compared with the pixel in positive and negative direction. The next following step will be the preservation and suppression of the edge pixel. If the magnitude of pixel $\mathrm{M}[\mathrm{i}, \mathrm{j}]$ is bigger than the two interpolation results on the gradient direction, it will be marked as candidate-edge point, otherwise it will be marked as non-edge point. Candidate-edge point will be preserved, while the non-edge point is suppressed or removed.

To get the clear picture of non-maximum suppression, further clarification is illustrated in the following figure (Figure 2).
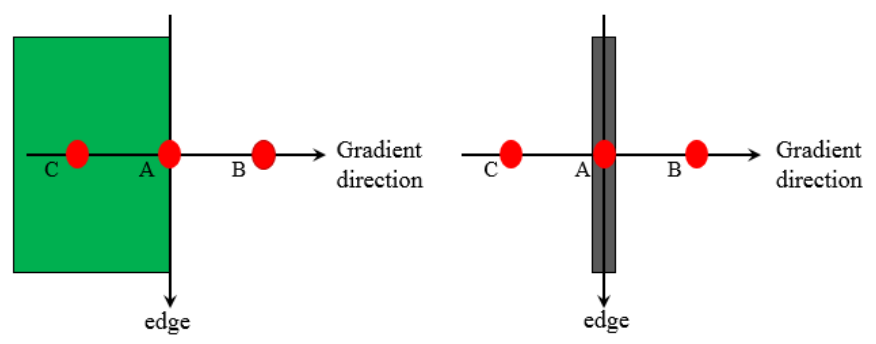

Figure 1 Non-maximum suppression process

Figure 1 shows that every pixel is checked when there is a local maximum in its neigborhood following the gradient direction. Point A is marked on the edge (vertical direction). The gradient direction is normal to edge, where point $\mathrm{B}$ and $\mathrm{C}$ lies. At this substage, point $\mathrm{A}$ is checked with point $\mathrm{B}$ and $\mathrm{C}$ to make sure whether it forms a local maximum, by using moving directional mask (green-shaded region). If the local maximum exists or formed, the particular edge pixel will be considered for next stage and preserved (candidate-edge point), else, it is suppressed to zero (non-edge point) (Mordvintsev \& $\mathrm{K}$, 2013). In a nutshell, the result that was obtained is a set edge points in the form of binary image, which is also being referred to as "thin edges".

\section{Checking and connecting edges}

The Canny algorithm adopts double-threshold method to select edge points after carrying on non-maximum suppression. The pixels whose gradient magnitude is above the high-threshold will be marked as edge points, and those whose gradient magnitude is under the lowthreshold will be marked as non-edge points, and the rest will be marked as candidate edge points. Those candidate edge points who are connected with edge points will be marked as edge points.

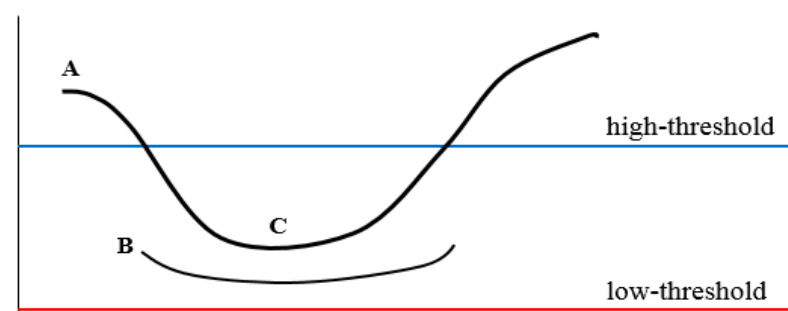

Figure 2 Double-threshold selection stage

Figure 2 shows the diagram of the pixels selection using doublethreshold (Mordvintsev \& K, 2013). As illustrated in the figure, the point $\mathrm{A}$ is above the high-threshold, thus it is marked as edge-point. Point $\mathrm{C}$ is below high-threshold, however it is connected to point $\mathrm{A}$. So, point $\mathrm{C}$ will also be marked as edge point. Different to point $\mathrm{B}$, it is not linked with any of edge points, atlhough it is located with same region of point $\mathrm{C}$. Therefore, point $\mathrm{B}$ is discarded. In short, the proper selection of high-threshold and low-threshold is important the ensure the correct result.

This process of selecting true edges from weak edges is named as hysteresis. This method reduces the influence of noise on the edge of the final edge image.

\section{The defect of the traditional Canny operator}

Canny edge detection heavily relies on the threshold values and Gaussian filter parameter, $\sigma$. This parameter $(\sigma)$ decides the size of the Gaussian filter. Greater value of $\sigma$ means bigger size of Gaussian filter or vice versa. Greater filter size caused image to be much more blurry. The choice of smaller $\sigma$ will be better for edge detection, but in return, less noise being removed. The choice of appropriate value of $\sigma$ is become quite difficult since it depends on the user selection. Most of the study done compare the performance of edge detection using a fixed parameter, but with different images. So, the effectiveness of the results obtained is limited.

\section{The proposed algorithm}

\section{A. Non-local means (NLM) filtering}

The NLM algorithm was pioneered by Buades and his colleagues in 2005. They introduced a novel approach by evaluating the similarity between two pixels $\mathrm{x}$ and $\mathrm{y}$, not only done by the intensity, but also by the difference of intensity in whole spatial neighborhood (Buades et al., 2005).

NLM's algorithm is designed in a way that a discrete noisy image $=\{v(i) \mid i \in I\}$, the estimated $N L[v](i)$, for a pixel $i$, is computed as a weighted average of all the pixels in the image,

$$
N L[v](i)=\sum_{j \in 1} w(i, j) v(j)
$$

where $w(i, j)$ is the weight assigned to value $v(j)$ for restoring the pixel $i$. The weight $w(i, j)$ depends on the similarity between pixels $i$ and $j$, and satisfy the general conditions $0 \leq(i, j) \leq 1$ and $\sum_{j \in 1} w(i, j)=1$. Under this condition, the weight $w(i, j)$ evaluates the similarity between the intensities of the local neighborhoods (patches) $v\left(N_{i}\right)$ and $v\left(N_{j}\right)$ centered on pixels $i$ and $j$. Mathematically, the weight can be defined by:

$$
w(i, j)=(1 / Z(i)) \exp \left(-\left\|v\left(N_{i}\right)-v\left(N_{j}\right)\right\|_{2, \sigma}^{2} / h^{2}\right)
$$

with normalization;

$$
Z(i)=\sum_{j} \exp \left(-\left\|v\left(N_{i}\right)-v\left(N_{j}\right)\right\|_{2, \sigma}^{2} / h^{2}\right)
$$

In the weight and normalization equation, the parameter $h$ controls the decay of the weights and is usually related to the level of noise in the image. Thus, a natural selection of $h$ will be of the form $h=c . \sigma$ where $c$ is a scalar and $\sigma$ is the level of noise in the image (Buades et al., 2005).

Buades' filter systematically uses all possible self-predictions the image can serve and utilizes the similarity concept of local patches to determine the pixel weights. As the reduction in patch size equal to one pixel, NLM filter becomes equivalent to the bilateral filter. Under stationarity assumptions, as the size of the image grow, there will be many similar patches to be found in all image details (Buades et al., 2005; Kumar, 2013). In summary, the NLM filter is effective at removing noise and smoothing the edges without losing too many fine structure and details. This pre-processing step is taken to reduce the high frequency components before the differentiation step.

\section{B. The proposed edge detection algorithm (Global-Canny)}


The term global is also known as the non-local component. Global Canny is referred to the hybrid NLM filter and Canny edge detection based algorithm. Figure 3 shows the proposed flowchart of Global Canny algorithm. In this paper, we propose a modification of Canny operator in order to better detect edges, particularly for MR image phantom application. MR images are degraded by Rician noise (Kumar, 2013). The first step in Canny operator is to apply Gaussian filter in order to reduce its sensitivity to noise. Problem is that it also smooths the edges. We propose NLM filter instead of Gaussian filter.

In our proposed method, the first step is to remove Rician noise using NL-means filter. Most of the major denoising methods available comprising Gaussian filtering, Wiener filtering and wavelet thresholding make assumptions about the image which can results in blurring and the loss of details. On the contrary, non-local means did not make the same assumptions. Instead, it assumes the image contains an extensive amount of self-similarity. Using this approach, non-local means filtering is able to remove noise and preserving image details. So, the improved part that was introduced here is the replacement of Gaussian smoothing with non-local means filtering.

After executing non-local means filtering, the original Canny operator from the second step is applied.

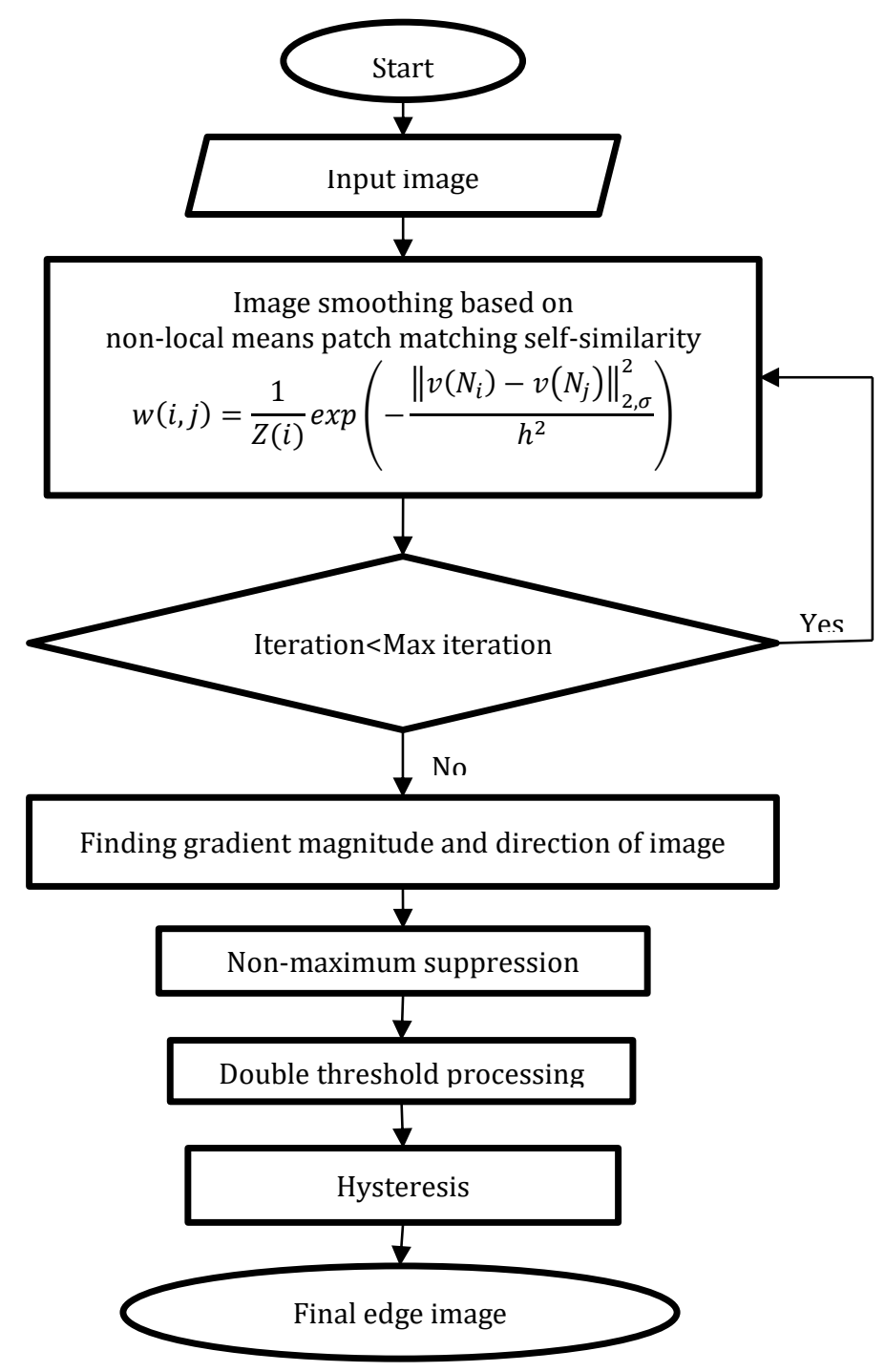

Figure 3 Flowchart of the methodology for the proposed algorithm

\section{RESULTS AND DISCUSSION}

\section{Experimental results}

The proposed method for edge detection in MR images of brain was implemented in Matlab R2012a. Experiments were performed on the platform Intel(R) Core(TM) i3-2310M CPU at 2.10GHz, 6GB RAM, Windows 7 Ultimate OS.

In order to test our proposed method, MR image phantom of kidney was used. The experimental results were organized in similar way as in (H. Chai et al., 2012), but the mentioned paper used phantom ultrasound image. The image used in this paper is shown in Figure 2(a) and is publicly available at YEZITRONIX Group Automation \& Control Industries Inc. website. MR image phantom acquired was first being read into MATLAB workspace.
Figure 5 shows edge detection in test image shown in Figure 4(a) by our proposed method. It was tested at different threshold values in order to determine the optimal one. In Figure 5, Canny operator are tested at threshold $\mathrm{T}=0.05,0.10,0.15$, and $\mathrm{T}=0.20$ with standard deviation at $\sigma=0.2,0.4,0.6$ and $\sigma=0.8$ respectively, for both original and new proposed method. In the first attempt, the threshold and standard deviation is set at 0.05 and 0.2 respectively, as shown in Figure 5 (a). Many of noisy parts were recognized as edges with this parameter values especially in original Canny algorithm. However, less noise is accepted as edges when using our proposed method. 


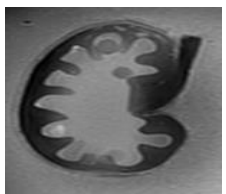

(a) Original $\mathrm{MR}$ image phantom

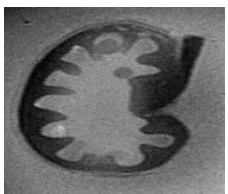

(b) Simulated noisy image

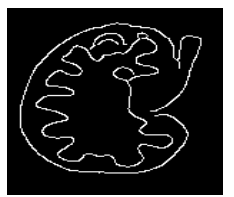

(c) Ground truth of MR image phantom

In the next following trials, we increased both threshold and sigma values. When we set $\mathrm{T}=0.10$ and $\sigma=0.4$, the image (Figure 5 (b)) exhibits a little less false edges but it is still not good enough to get the desired result. The presence of noise once again cause edge misdetection in most of area in the image using original Canny operator. The image shows better edge detection using our proposed method, but some noise was regarded as edges and some parts of phantom image lines eroded.

Another trial had been done to improve the result by increasing the parameter values to $\mathrm{T}=0.15$ and $\sigma=0.6$. Using this setting, better edge detection were performed as shown in Figure 5(c). Our proposed method convey better edge detection as compared to the other method. There was some noise was still recognized as edges but this occurrence was significantly lower than that in the previous attempt.

\section{Traditional} Canny
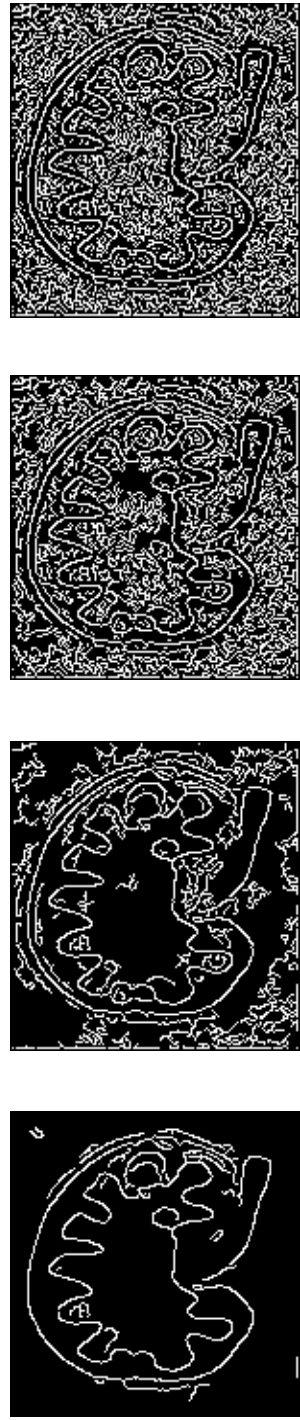

Anisotropic Diffusion (AD)- Canny
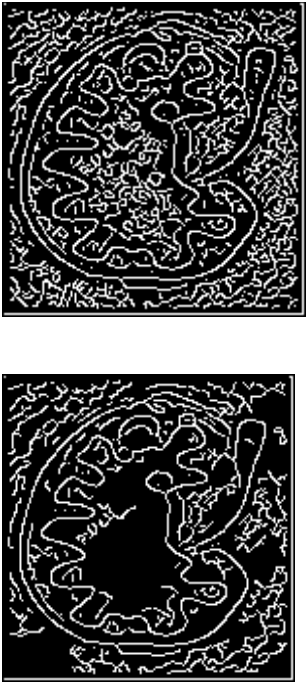

(a)
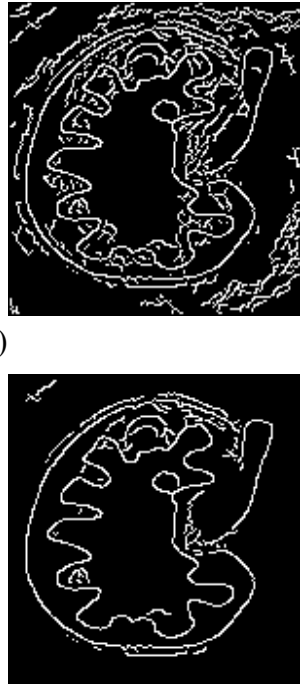

(b)
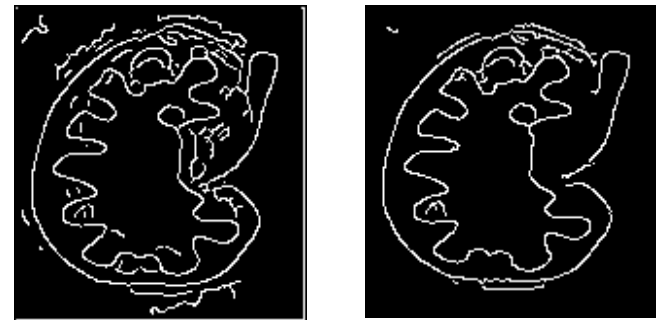

(c)
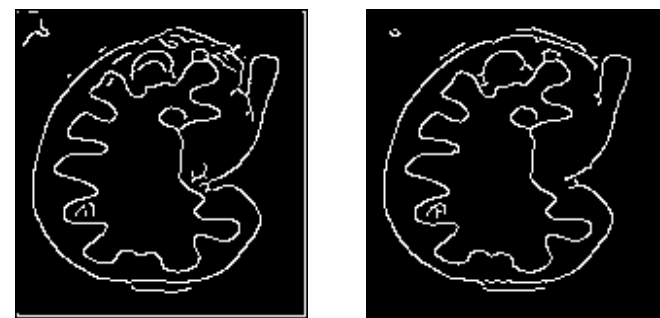

In the final attempt, Figure 5 (d) shows much better edge detection. The parameter values were set at $\mathrm{T}=0.20$ and $\sigma=0.8$. Obviously, our proposed method shows excellent detection as compared to the other algorithm with precise and connected edge lines. In summary, the Global Canny had minimized the probability of false edges caused by noise, as well as missing real edges. Second, the proposed method show edges detected is as close as possible to the true edges. Third, the detector return one point only for each true edge point.

\section{Performance evaluation}

The experimental results are further evaluated based on quantitative analysis. The performance of algorithm are evaluated similarly in (Deng et al., 2013) and (Deng et al., 2013). Since noise reduction and edge preservation are conflicting objectives, thus the performance assessment require simultaneous comparison. In this part, quantitative parameter such the Peak Signal to Noise Ratio (PSNR), image entropy, average gradient, the correlation coefficient and the similarity percentage are being quantified (Deng et al., 2013). However, only output image using optimum parameter are being taken into consideration.

\section{Bilateral- \\ Canny \\ Proposed method}
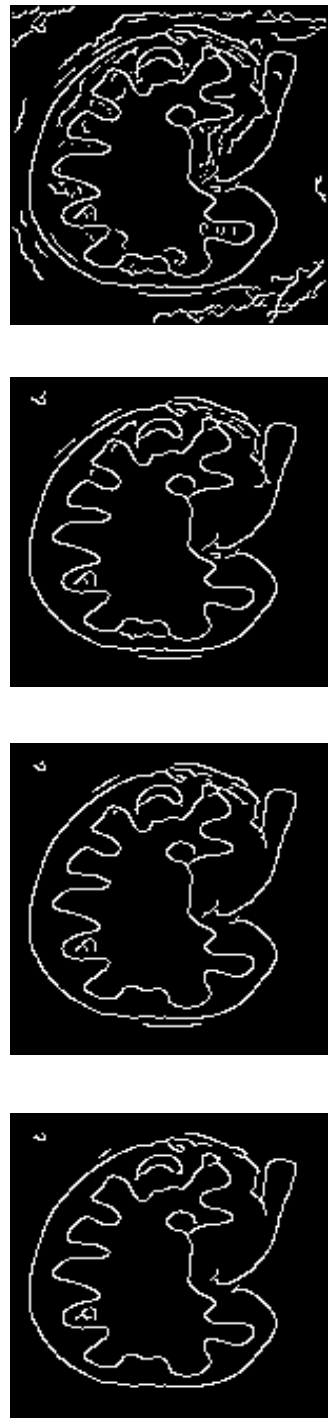

(d)

Figure 5 Comparison between traditional Canny with other Canny-based methods with double thresholding parameter setting 
The PSNR is the parameter that evaluates the performance of image enhancement algorithm by comparing the enhanced image with the original input image. The formula computes the ratio between the maximum possible value of a signal and the power of the noise signal, which is given as follows:

$$
P S N R=10 \log _{10}\left(255^{2} / M S E\right)
$$

where the MSE is the Mean Squared Error and is given by the following equation:

$$
M S E=\frac{1}{m n} \sum_{0}^{m-1} \sum_{0}^{n-1}\|f(i, j)-g(i, j)\|^{2}
$$

with $f$ and $g$ are referring the original input image and the processed image, respectively. In fact, $M S E$ compare the "true" pixel values of original input image the processed output image. High quality image is defined by higher value of PSNR which means $M S E$ must be lower.

The image entropy taking average of the information content in an image to encode the image values. Higher value image entropy indicates higher amount of information existed in an image. The calculation of image entropy yields the value of histogram dispersion. The image entropy, $H$ is given by:

$$
H=-\sum_{i=0}^{L-1} P_{i} \cdot \log _{2}\left(P_{i}\right)
$$

where $P_{i}$ is the probability of two adjacent pixels difference (grey values) is equal to $i$, and $i$ is the pixel intensity of an image.

The Pearson Correlation coefficient measure the likeliness of the original and the processed image. Higher value of correlation indicates that the output image is much closer to input image which has the maximum value of 1 . Correlation of two images can be computed by using the following formula:

$$
\operatorname{Corr}_{1}=\frac{\sum_{i}\left[\left(x_{i}-x_{m}\right)\left(y_{i}-y_{m}\right)\right]}{\sqrt{\sum_{i}\left(x_{i}-x_{m}\right)^{2}} \sqrt{\sum_{i}\left(y_{i}-y_{m}\right)^{2}}}
$$

with magnitude of $x_{m}$ and $y_{m}$ is defined as:

$$
\begin{aligned}
& x_{m}=\frac{1}{m n} \sum_{i=0}^{m-1} \sum_{j=0}^{n-1} x_{i} \\
& y_{m}=\frac{1}{m n} \sum_{i=0}^{m-1} \sum_{j=0}^{n-1} y_{i}
\end{aligned}
$$

In the above equation $x_{i}$ is denoted as the intensity of the $i^{\text {th }}$ pixel in image $1, y_{i}$ is the intensity of the $i^{\text {th }}$ pixel in image $2, x_{m}$ is the mean intensity of image 1 , and $y_{m}$ is the mean intensity of image 2 .

Image clarity measure the structural quality that reflect the change in image texture and details. Better image quality has higher value of the image clarity. This parameter can be expressed using this following equation:

$$
\nabla \bar{G}=\frac{1}{m n} \sum_{i=1}^{M} \sum_{j=1}^{N} \sqrt{\left[\left(\frac{\partial f(i, j)}{\partial x}\right)^{2}+\left(\frac{\partial f(i, j)}{\partial y}\right)\right]}
$$

where $\partial f(i, j) / \partial x$ and $\partial f(i, j) / \partial y$ are one-order differential of pixel $(i, j)$ in $\mathrm{x}$ and $\mathrm{y}$ direction respectively.

Table 1 show the performance comparison between Global Canny with other Canny based algorithm.
Table 1 Performance evaluation of traditional Canny, anisotropic diffusion-Canny, Bilateral-Canny and Global-Canny-based method

\begin{tabular}{|l|l|l|l|l|l|}
\hline $\begin{array}{l}\text { Evaluation } \\
\text { parameter }\end{array}$ & $\begin{array}{l}\text { Ground } \\
\text { truth } \\
\text { image }\end{array}$ & $\begin{array}{l}\text { Traditio- } \\
\text { nal } \\
\text { Canny }\end{array}$ & $\begin{array}{l}\text { AD- } \\
\text { Canny }\end{array}$ & $\begin{array}{l}\text { Bi- } \\
\text { lateral } \\
\text { Canny }\end{array}$ & $\begin{array}{l}\text { Global } \\
\text { Canny }\end{array}$ \\
\hline PSNR & - & 25.669 & 25.742 & 25.671 & 25.667 \\
\hline Entropy & 6.9533 & 6.9804 & 6.9114 & 6.8489 & 6.89 \\
\hline $\begin{array}{l}\text { Image } \\
\text { clarity }\end{array}$ & 47.30 & 54.36 & 44.79 & 33.09 & 33.35 \\
\hline Correlation & 1 & 0.989 & 0.965 & 0.991 & 0.993 \\
\hline $\begin{array}{l}\text { Similarity } \\
\text { percentage } \\
(\%)\end{array}$ & 100 & 73.67 & 74.02 & 82.47 & 87.22 \\
\hline
\end{tabular}

In term of noise reduction, anisotropic diffusion-Canny perform the best with the highest PSNR value. This was followed by bilateral Canny, traditional Canny and lastly global Canny. The anisotropic diffusion-Canny method outstands traditional Canny and other method as well. However, the filter downfall is that it also remove image detail which cause blurry image representation. The image quality is not preserved and complicate the correct edge detection process.

Image texture and fine details is the most prominent using traditional Canny method. This can be seen in the high image entropy and image clarity value. However, it is possible that the method was influenced by the existence of the noise. This cause edge misdetection to be occurred. This problem happens when the noisy edges dominate the process and causes the miss for valid edges while creating noiseinduced false edges.

Image correlation is important in finding the degree of similarity between the input and the processed image. The proposed method exhibits the highest correlation as compared to others. It shows that the proposed method successfully remove noise while preserving image properties, as the processed image is very close to the input phantom image. This statement is supported by the highest percentage difference shown using the proposed method, by comparing the final edge detection with the synthesized ground truth.

\section{CONCLUSION}

In this paper, we proposed an algorithm based on Canny operator for edge detection in Magnetic Resonance phantom image of internal organ. The new method was incorporated NLM filter instead of using Gaussian filter in Canny operator. Experimental results have shown that with the modified Canny operator, edges in noisy Magnetic Resonance phantom image can be recognized very successfully. Future works may involve modification of the original algorithm for different application of internal organs, different type of medical images and different type of noise.

\section{ACKNOWLEDGEMENT}

The authors gratefully acknowledge the research grant provided to this study by Research Management Centre sponsored by Ministry of Education, Malaysia. Vot: $16 \mathrm{H} 19$ (Research University Grant), Universiti Teknologi Malaysia, Johor Bahru, Malaysia.

\section{REFERENCES}

Agaian, S., Almuntashri, A., Papagiannakis, A. T. (2009). An improved Canny edge detection application for asphalt concrete. 2009 IEEE International Conference on Systems, Man and Cybernetics, 1-14 October 2009. San Antonio, TX, USA: IEEE, 3683-3687.

Mukherjee, A., Kundu, D. (2013). Motion analysis in video surveillance using edge detection techniques. IOSR Journal of Computer Engineering (IOSRJCE) , 12(6), 10-15.

Aslam, A., Khan, E., Sufyan Beg, M. (2015). Improved edge detection algorithm for brain tumor segmentation. Procedia - Procedia Computer Science, 58, 430-437. 
Bandyopadhyay, S. K. (2012). Edge detection from CT images of lung. International Journal Of Engineering Science \& Advanced Technology, 2(1), 34-37.

Benson, E. R., Reid, J. F., Zhang, Q. (2003). Machine vision-based guidance system for agricultural grain harvesters using cut-edge detection. Biosystems Engineering, 86(4), 389-398.

Buades, A., Coll, B., Morel, J.-M. J.-M. (2005). A non-local algorithm for image denoising. 2005 IEEE Computer Society Conference on Computer Vision and Pattern Recognition (CVPR'05). 20-25 June 2005. San Diego, CA, USA, USA: IEEE, 60-65.

Canny, J. (1986). A computational approach to edge detection. IEEE Transactions on Pattern Analysis and Machine Intelligence, 8(6), 679698.

Chai, H., Wee, L. K., Supriyanto, E. (2012). Edge detection in ultrasound images using speckle reducing anisotropic diffusion in canny edge detector framework. WSEAS Transactions on Biology and Biomedicine, $8(2), 51-60$.

Chai, H. Y., Wee, L. K., Swee, T. T., Hussain, S. (2011). Gray-level cooccurrence matrix bone fracture detection. WSEAS Transactions on Systems, 10(1), 7-16.

Chai, H. Y., Wee, L. K., Swee, T. T., Salleh, S. H. (2011). Adaptive crossed reconstructed (ACR) Kmean clustering segmentation for computeraided bone age assessment system. International Journal of Mathematical Models and Methods in Applied Sciences, 5(3), 628-635.

Shubhangi, D. C., Chinchansoor, R. S., Hiremath, P. (2012). Edge detection of femur bones in X-ray images - A comparative study of edge detectors International Journal of Computer Applications, 42(2), 975-8887.

Deng, C., Wang, G., Yang, X. (2013). Image edge detection algorithm based on improved canny operator. 2013 International Conference on Wavelet Analysis and Pattern Recognition, Tianjin, 14-17 July, 2013 (14-17).

Dhankhar, P., Sahu, N. (2013). A review and research of edge detection techniques for image segmentation. International Journal of Computer Science and Mobile Computing, 2(July), 86-92.

Giuliani, D. (2012). Edge detection from MRI, DTI images with an anisotropic vector field flow using a divergence map. Algorithms, 5(4), 636-653.

Gudbjartsson, H., Patz, S. (1995). The rician distribution of noisy mri data. Magnetic Resonance in Medicine, 34(6), 910-914.

Gupta, R. (2016). Enhanced edge detection technique for satellite images. International Conference on Cloud Computing and Security. 29-31 July 2016. Nanjing, China : Springer, 273-283.

Hou, X., Dong, Y., Zhang, H., Gu, J. (2009). Application of a self-adaptive Canny algorithm for detecting road surface distress image. 2009 Second International Conference on Intelligent Networks and Intelligent Systems. 1-3 November 2009. Tianjin, China: IEEE, 354-357.

Huertas, A., Medioni, G. (1986). Detection of intensity changes with subpixel accuracy using Laplacian-Gaussian masks. IEEE Transactions on Pattern Analysis and Machine Intelligence, PAMI-8(5), 651-664.

Jena, K. K., Mishra, S., Mishra, S. (2015). Edge detection of satellite images: A comparative study. International Journal of Innovative Science, Engineering \& Technology, 2(3), 75-79.

Jia, J. (2009). A machine vision application for industrial assembly inspection. 2009 Second International Conference on Machine Vision. 28-30 December 2009. Dubai, United Arab Emirates: IEEE, 172-176.

Jia, X. (2010). Fabric defect detection based on open source computer vision library OpenCV. In 2010 2nd International Conference on Signal Processing Systems. 5-7 July 2010. Dalian, China: IEEE, 342-345.

Karamiani, A., Farajzadeh, N. (2014). Detecting and tracking moving objects in video sequences using moving edge features. Scientific Cooperations International Workshops on Electrical and Computer Engineering Subfields, (August), 88-92.

Khadse, M. V, Kale, N. S. (2016). An effective object detection video surveillance and alert system. IJCA Proceedings on National Conference on Advances in Computing, Communication and Networking . 18-22 June 2016. Jalgaon, Maharashtra, India: IJCA, pp. 18-21.

Kumar, B. K. S. (2013). Image denoising based on non-local means filter and its method noise thresholding. Signal, Image and Video Processing, 7(6), 1211-1227.

Lakhani, K., Minocha, B., Gugnani, N. (2016). Analyzing edge detection techniques for feature extraction in dental radiographs. Perspectives in Science, 8(4), 395-398.

Lakshmi, S., Sankaranarayanan, D. V. (2010). A study of edge detection techniques for segmentation computing approaches. International Journal of Computer Applications, CASCT(1), 35-41.

Li, E. Sen, Zhu, S. L., Zhu, B. S., Zhao, Y., Xia, C. G., Song, L. H. (2009). An adaptive edge-detection method based on the Canny operator. Proceedings - 2009 International Conference on Environmental Science and Information Application Technology, ESIAT 2009, 1(3). 4-5 July 2009. Wuhan, China: IEEE, 465-469.

Mordvintsev, A., K, A. (2013). Canny edge detection - OpenCV-Python
Tutorials 1 documentation. Retrieved September 25, 2017, from http://opencv-python-

tutroals.readthedocs.io/en/latest/py_tutorials/py_imgproc/py_canny/py_ca nny.html

Muthukrishnan, R., Radha, M. (2011). Edge detection techniques for MRI Brain Image Segmentation. International Journal of Computer Science and Information Technology, 3(6), 259-267.

Nercessian, S. C., Agaian, S. S., Panetta, K. A. (2009). A generalized set of kernels for edge and line detection. Proceedings of SPIE, 7245. San Jose, California, United States, 1-12.

Nguyen, T. B., \& Ziou, D. (2000). Contextual and non-contextual performance evaluation of edge detectors. Pattern Recognition Letters, 21(9), 805-816.

Nikolic, M., Tuba, E., Tuba, M. (2016). Edge detection in medical ultrasound images using adjusted canny edge detection algorithm. IEEE Transactions on Image Processing, 0-3.

Pal, N. R., Pal, S. K. (1993). A review on image segmentation techniques. Pattern Recognition, 26(9), 1277-1294.

Pellegrino, F. A., Vanzella, W., Torre, V. (2004). Edge detection revisited. IEEE Transactions on Systems, Man, and Cybernetics, Part B. Cybernetics, 34(3), 1500-1518.

Pietikainen, M., Okun, O. (2001). Edge-based method for text detection from complex document images. Proceedings of Sixth International Conference on Document Analysis and Recognition. 13 September 2001. (Seattle, WA: IEEE, 8-13.

Punarselvam, E., Suresh, P. (2011). Edge detection of CT scan spine disc image using canny edge detection algorithm based on magnitude and edge length. 3rd International Conference on Trendz in Information Sciences \& Computing (TISC2011). 8-9 December 2011. Chennai, India: IEEE, 136140.

Qiu, K., Sun, K., Ding, K., Shu, Z. (2016). A fast and robust algorithm for road edges extraction from lidar data. International Archives of the Photogrammetry, Remote Sensing and Spatial Information Sciences ISPRS Archives, 41(July), 693-698.

Rumheller, D. M. (1993). General Expressions for Rician Density and Distribution Functions. IEEE Transactions on Aerospace and Electronic Systems, 29(2), 580-588.

Savant, S. (2014). A review on edge detection techniques for image segmentation. International Journal of Computer Science and Information Technologies, 5(4), 5898-5900.

Selvakumar, P., Hariganesh, S. (2016). The performance analysis of edge detection algorithms for image processing. 2016 International Conference on Computing Technologies and Intelligent Data Engineering (ICCTIDE'16). 7-9 January 2016. Kovilpatti, India: IEEE, 1-5.

Sijbers, J., den Dekker, A. J., Scheunders, P., Van Dyck, D. (1998). Maximumlikelihood estimation of Rician distribution parameters. IEEE Transactions on Medical Imaging, 17(3), 357-61.

Sirmacek, B., Unsalan, C. (2010). Road network extraction using edge detection and spatial voting. In International Conference on Pattern Recognition (pp. 3117-3120)

Voorhees, H., Poggio, T. (1987). Detecting textons and texture boundaries in natural image. Proceedings of the First International Conference on Computer Vision London. 8-11 June 1987. London: IEEE, 250-258.

Wang, B., Fan, S. (2009). An improved Canny edge detection algorithm. 2009 Second International Workshop on Computer Science and Engineering. 28-30 October 2009. Qingdao, China: IEEE, 497-500.

Yu, C., Song, Y., Meng, Q., Zhang, Y., Liu, Y. (2015). Text detection and recognition in natural scene with edge analysis. IET Computer Vision, 9(4), 603-613.

Zhang, B., Huang, W., Gong, L., Li, J., Zhao, C., Liu, C., Huang, D. (2015) Computer vision detection of defective apples using automatic lightness correction and weighted RVM classifier. Journal of Food Engineering, $146,143-151$. 